\title{
Sebastian Tengnagel und Johann Seyfried Österreichische Geschichtsschreibung zWischen SpÄthumanismus und Gegenreformation
}

\section{Thomas Wallnig}

In den Jahrzehnten um 1600 fragmentierte sich die humanistische Gelehrtenrepublik. Die politische und konfessionelle Indienstnahme von Wissen machte in einem stärkeren Maß als zuvor aus gelehrten Kollegen politische Feinde, für die das Aufrechterhalten irenischer Grundhaltungen zunehmend mehr Aufwand bedeutete und Energie erforderte. Mittelfristig trug diese Situation zur Formierung „neuer” Wissensparadigmen bei, die auch der längerfristigen politischen Einteilung Europas in vermeintlich „progressive” und „konservative” Regionen Vorschub leisteten. In den Jahrzehnten um 1600 begannen die Grundlinien dieses Bruches sichtbare Konturen anzunehmen.

Die folgenden Seiten berichten von Bekanntschaft und Briefwechsel zweier gleichsam nebenberuflicher Gelehrter, die zur fraglichen Zeit unterschiedliche Ausprägungen intellektueller Kultur im sukzessive rekatholisierten Zentraleuropa repräsentierten. Johann Seyfried war Abt des niederösterreichischen Zisterzienserstiftes Zwettl und dortselbst ein aktiver Verfechter habsburgischer Rekatholisierungspolitik. Zugleich plante Seyfried die Herausgabe einer österreichischen Geschichte unter dem Titel „Annales Austriae”. Sebastian Tengnagel war kaiserlicher Bibliothekar in Wien und verantwortlich für die Verwaltung, Erweiterung, Verzeichnung und Verfügbarmachung der Bestände der Hofbibliothek. Tengnagel interessierte sich besonders für orientalische Handschriften, die er auch privat sammelte. ${ }^{1}$

$1 \quad$ Die Forschungen zu Tengnagel und Seyfried stehen im Zusammenhang mit dem vom FWF geförderten Projekt P-30511, „The Oriental Outpost of the Repubilc of Letters": https://geschichtsforschung.univie.ac.at/forschung/ oorpl/ [21.02.2020]. 
In einem der drei Bände der Tengnagel-Korrespondenz, die in der Österreichischen Nationalbibliothek verwahrt werden, finden sich sieben Schreiben Seyfrieds an Tengnagel aus den Jahren 1621 und 1622. Bereits ein Jahrzehnt davor bestand jedoch Kontakt zwischen den beiden Männern, wie die Beteiligung des Hofbibliothekars an der wichtigsten Publikation Seyfrieds zeigt. Es handelte sich dabei um eine fiktive Genealogie, die die Habsburger mit einer stadtrömischen Familie in Verbindung brachte. Auf den folgenden Seiten werden diese Materialien herangezogen, um die beiden Gelehrten und ihr Verhältnis zueinander zu charakterisieren. Zugleich sollen aber auch die wesentlichen Themenfelder ihres Austauschs charakterisiert und in einem breiteren wissens- und wissenschaftsgeschichtlichen Kontext verortet werden.

Betrachtet man die beiden Biographien, so fällt auf, dass Seyfried ebenso wenig wie Tengnagel einen österreichischen Hintergrund hatte. Seyfried (1577-1625) stammte - wie übrigens auch Kardinal Melchior Khlesl - aus Breslau (Wrocław), einem der späthumanistischen Zentren Zentraleuropas im ausgehenden 16. Jahrhundert. ${ }^{2}$ Seyfried erhielt dortselbst ein Kanonikat, das Domkapitel sandte ihn nach Rom ans Collegium Germanicum und später trat er in Frankreich in den Zisterzienserorden ein. Er kam sodann nach Heiligenkreuz in Niederösterreich und wurde von dort als Abt nach Zwettl postuliert. ${ }^{3}$

In dieser Rolle zählte er zu jenen Prälaten, die sich die Agenda der Rekatholisierung in enger Zusammenarbeit mit den weltlichen und geistlichen Autoritäten besonders angelegen sein ließen. Dies äußerte sich in Seyfrieds Rolle als ständischer Verordneter und geheimer Gesandter für Kaiser Matthias ebenso wie in seiner Funktion als

Breslau wird in seiner Rolle als intellektuelles Zentrum Zentraleuropas umfänglich vorgestellt bei Gábor Almási, The Uses of Humanism. Johannes Sambucus (1531-1584), Andreas Dudith (1533-1589), and the Republic of Letters in East Central Europe, Leiden 2009.

3 Zu Seyfried vgl. Charles de VISCH, Bibliotheca scriptorum sacriordinis Cisterciensis, Köln 1656, 229; Florian WATzL, Die Cistercienser von Heiligenkreuz. Graz 1898,60 . 
Generalvisitator seines Ordens in Österreich, Böhmen, Mähren, Schlesien und der Lausitz sowie seiner Aktivität im Zusammenhang mit seinem Kloster selbst: Zwettl war Schauplatz und Opfer der kriegerischen Auseinandersetzungen in der ersten Phase des Dreißigjährigen Krieges. ${ }^{4}$ Seyfried war freilich - ebenso wie beispielsweise der Benediktiner Kaspar Plautz von Seitenstetten - einer jener Verfechter der vortridentinischen Orden, die vehement deren Position in der Gegenreformation zu behaupten suchten. Das erklärt auch, warum Seyfried sich etwa in rechtlichen Fragen mit der Gesellschaft Jesu auseinandersetzte, beispielsweise im Hinblick auf Zwettler Rechte am Nonnenkloster St. Bernhard bei Horn.

Aus einem gänzlich anderen Teil Europas, der jedoch ebenso wie Schlesien zum Austragungsort religiöser Kontroversen wurde, stammte Sebastian Tengnagel. Er wurde 1573 in Buren in der Grafschaft Geldern geboren. Dieser Teil der Grafschaft schloss sich 1579 jenen Provinzen an, die sich von der habsburgisch-spanischen Herrschaft losgesagt und somit den Unabhängigkeitskrieg der Vereinigten Provinzen initiiert hatten. Tengnagel studierte in Heidelberg, einem der Zentren kalvinistisch geprägter, doch interkonfessioneller späthumanistischer Gelehrsamkeit, und graduierte später wohl in Italien zum Doktor beider Rechte. Um 1598 wurde er von seinem Landsmann Hugo Blotius, der damals der kaiserlichen Bibliothek vorstand, nach Wien geholt, wo er als dessen Gehilfe tätig war. Tengnagel folgte Blotius nach dessen Tod $1608 \mathrm{im}$ Amt und heiratete seine Witwe, die durch ihre Abkunft aus einer Wiener Familie die Integration in die städtische Gemeinschaft erleichterte.

Tengnagel knüpfte an die drei Sammlungsschwerpunkte seines Vorgängers an und sammelte $\mathrm{zu}$ österreichischen und kirchengeschichtlichen Fragen sowie zur Geschichte der orientalischen Kulturen, womit im Verständnis der Zeit vornehmlich jene arabische,

4 Die Vorgänge sind detailliert geschildert in: Andreas FidLer, Geschichte der ganzen österreichischen, klösterlichen und weltlichen Klerisey, 9: Die k.k. Haupt-und Residenzstadt Wien, Wien 1788, 84-91. 
persische und osmanisch-türkische gemeint war. Er betätigte sich als Quellenlieferant für andere Gelehrte, besonders jene, die eine katholische bzw. kaiserfreundliche Lesart der Kirchengeschichte verfochten. Ein Beispiel hierfür ist der Jesuit Jakob Gretser, der hinter Tengnagels einziger gedruckter Publikation, den „Vetera monumenta contra schismaticos" stand. ${ }^{5}$ Es handelte sich um einen Baustein in der lange, erbittert und im Nachklang der Reformation nochmals intensiviert geführten Kontroverse um das Verhältnis von kirchlicher und weltlicher Autorität im Reich. ${ }^{6}$

Zugleich zeigte Tengnagel als Bibliothekar durchaus Interesse und Sensibilität für die Prioritäten und Bedürfnisse seiner Sammlung. Er versuchte, wenn auch ohne Erfolg, das Amt eines österreichischen Klosterrates zu erlangen, jener Institution, die von Kaiser Maximilian II. eingerichtet worden war, um die finanzielle Gebarung von Klöstern überwachen zu können. Es ging hier um die Aufbesserung seines Gehalts, doch blickte Tengnagel auch in anderen Zusammenhängen konsequent und nicht ohne Eigeninteresse auf die Ressourcen religiöser Gemeinschaften wie Schotten und Minoriten.

Auch Seyfried war bereits vor seiner Postulation nach Zwettl in Heiligenkreuz während mehrerer Jahre als Bibliothekar tätig. Ein so entwickeltes Verständnis für die tagespolitische Relevanz historischer Quellen mag auch dazu geführt haben, dass Seyfried im Jahr 1608 Erzherzog Matthias anlässlich dessen ungarischer Krönung im Namen von Abt und Konvent ein Festgedicht „hieroglyphicis figuris" überreichte, das bereits den Zusammenhang zwischen den

5 Sebastian Tengnagel, Vetera monumenta contra schismaticos iam olim pro Gregorio VII. aliisque nonnullis pontificibus Romanis conscripta, Ingolstadt 1612. Die Verbindung zu Gretser wird in der Widmungsvorrede angesprochen.

6 Stefan Benz, Zwischen Tradition und Kritik. Katholische Geschichtsschreibung im barocken Heiligen Römischen Reich, Husum 2003, 80-90. 
Habsburgern und der stadtrömischen Gens herstellte.? Von Matthias zu klarstellender Erklärung aufgefordert, verfasste Seyfried eine umfassende Rechtfertigungsschrift. Diese, der "Anicianische Baum” („Arbor Aniciana”, siehe Авв. ı), legt in drei Büchern nicht nur die vermeintliche Abkunft der Habsburger von der stadtrömischen gens Anicia dar, ${ }^{8}$ sondern behauptet dasselbe auch für den Heiligen Benedikt, was gewissermaßen die regierende Dynastie und den Orden von Citeaux in ein genealogisch-spirituelles Naheverhältnis rücken sollte. ${ }^{9}$ Tengnagel wurde in diese wohl schon für zeitgenössische Ohren nicht ohne weiteres unproblematische Geschichtskonstruktion dahingehend involviert, dass er eine Approbation zu dem Werk verfasste. Er wusste darin potentiellen Zweifel herunterzuspielen und nahm zugleich in seiner Rolle als Hofbibliothekar quasi eine approbierende Rolle ein.

AuchSeyfried magbeiderAbfassungder „Arbor"klargewesensein, dass eine historiographische Reputation mit fiktiver Genealogie allein nicht zu gewinnen sein würde. So betonte er bereits in der Widmungsvorrede an Matthias die Bedeutung einer Urkundensammlung Rudolfs I., die ihm aus Heiligenkreuz bekannt war. Er verband diese Elemente zu einem umfassenderen Plan einer österreichischen Geschichte. ${ }^{10}$

Schon unter Leopold I. freilich war, wie Stefan Benz schreibt, genealogische Geschichtsschreibung nicht beliebt, was für Kaspar Schoppes „Stemma” ebenso galt wie für die Arbeiten seines Konkurrenten Seyfried: „Zu willkürlich und unseriös erschienen die

Johann Seyfried, Arbor Aniciana seu Genealogia serenissimorum augustissimae Austriae domus principum, 3 Bde., Wien 1613, Benevolo lectori; irrig dargestellt in der ansonsten anregenden Abhandlung Fabio Martelli, L'Arbor Aniciana di Joannes Seifried. Una teologia politica asburgica alla vigilia della guerra dei trent'anni, Bologna 2012, 7f.

8 Zur Gens: Elimar KLEBS, Anicius, in: Paulys Realencyclopädie der classischen Altertumswissenschaft, 1.2. Stuttgart 1894, col. 2196-2208.

9 Alphons Lнотsку, Die Wiener Palatina und die Geschichtsforschung zur Zeit Sebastian Tengnagels, in: Die Österreichische Nationalbibliothek. Festschrift für Joseph Bick, Wien 1948, 450-462; zur Korrespondenz zwischen Seyfried und Tengnagel siehe bes. 46lf.

10 Benz, Zwischen Tradition und Kritik (wie Anm. 6), 98. 


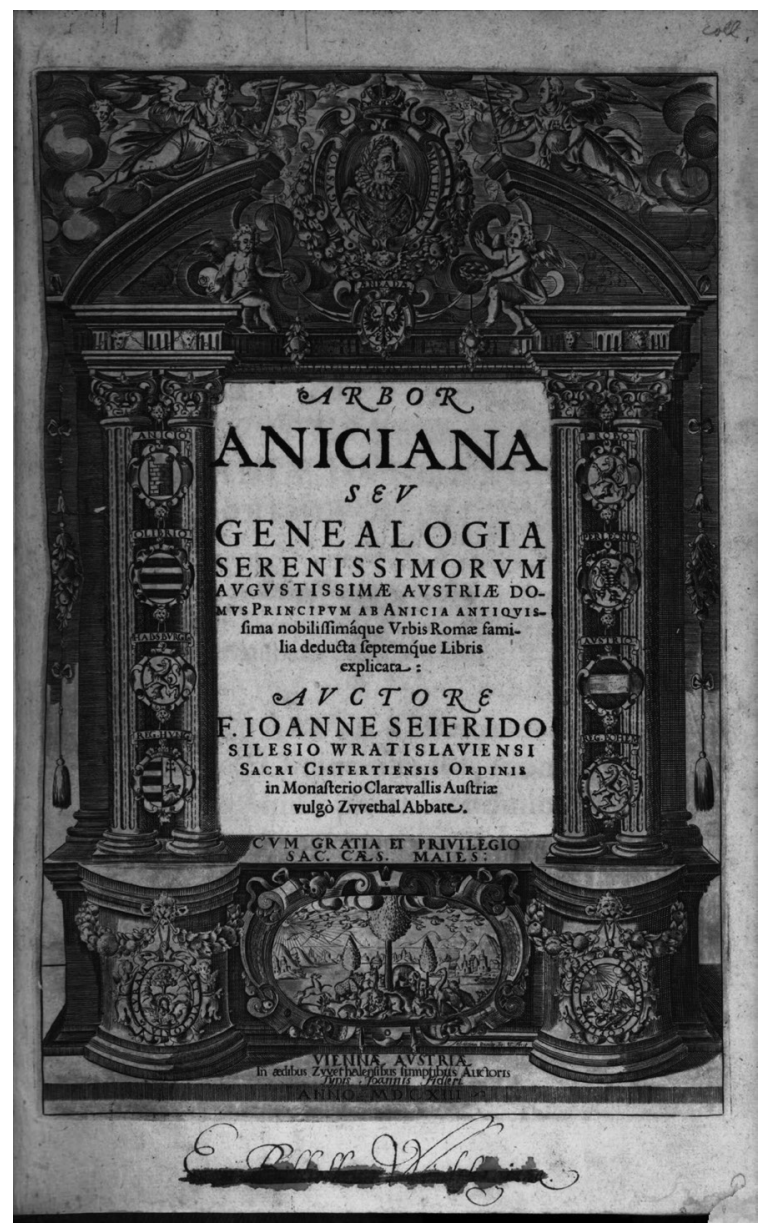

Abb. 1: Frontispiz der „Arbor Aniciana” von Johannes Seyfried (copyright: Österreichische Nationalbibliotek).

genealogischen Konstrukte, zu belastet durch ihre Konnotation von Unwissenschaftlichkeit war die blasse Erinnerung an die fabelhafte Hofhistoriographie Maximilians I., zu offensichtlich die Einsicht, 
daß beeindruckende Genealogien wohlfeil und damit an sich wertlos waren."11

Ein knappes Jahrzehnt lag nun zwischen der Publikation der „Arbor” und dem Auftakt des kurzen erhaltenen Briefwechsels zwischen Seyfried und Tengnagel im Jahr 1621. Man kann mutmaßen, dass es in den dazwischen liegenden Jahren Austausch und Kontakt gegeben hat, ebenso ist aber plausibel, dass Seyfried zumindest in den Jahren 16181621 tatsächlich vor allem mit den Folgen von Krieg und Verwüstung in Zwettl befasst war. Die Korrespondenz vermittelt jedenfalls den Eindruck, dass Seyfried 1621 die Arbeit an seiner "Österreichischen Geschichte" wieder aufnehmen und sich der Unterstützung des Hofbibliothekars versichern wollte. So kreisen die Themen der Korrespondenz in erster Linie um Bücher und Handschriften, zugleich aber auch um die Rolle der beiden Protagonisten im Hinblick auf Konzeption, Umfang, quellenmäßige Unterlegung und institutionelle Kompetenz.

Seyfried beklagt mehrmals den Krieg und seine zerstörerische und deprimierende Wirkung. In den Wunsch nach Frieden und Muße mischt sich jener nach einem Sieg des „Bayerischen Löwen” sowie die Abneigung gegen "die” Ungarn - „semichristianos semihominesque Hungaros”.12

Die deklarierte politische Parteinahme hatte jedoch auch einen sehr pragmatischen Hintergrund. Im April 1622 berichtet Seyfried davon, dass der "praefectus” seines Klosters, Bartholomäus Klimannus, im Zuge der Eroberung der Burg Vöttau auf eine ansehnliche Bibliothek aufmerksam geworden war: Wäre es nicht Tengnagels Aufgabe, die Bibliothek zu begutachten (,visere ac recensire")? $?^{13}$ Es wäre auch eine gute Gelegenheit für einen Besuch in Zwettl, zumal der fragliche Ort nahe ist, Benz, Zwischen Tradition und Kritik (wie Anm. 6), 408. Zur weiteren Bearbeitung der Briefsammlung Rudolfs I. im 18. Jahrhundert vgl. ebd., 454.

12 Seyfried an Tengnagel, 30. August 1621; Wien, Österreichische Nationalbibliothek (ÖNB), Cod. 9737s, 244r-245v. Vergleichbare Bemerkung 11. Februar 1621, ebd., 222r-v: pessima hac temporum confusione animus malorum asuetus.

13 Seyfried an Tengnagel, 20. April 1622, ÖNB Cod. 9737s, 267r-v. 
ebenso wie - so Seyfried mit vielsagender Andeutung - Krumau. Derselbe Balthasar Klimannus war schon im Herbst zuvor in ähnlicher Sache und ebenfalls im Auftrag des Abtes an Tengnagel herangetreten; damals ging es um die berühmte Rosenbergsche Bibliothek in Wittingau. ${ }^{14}$ Offenbar ließ sich Seyfried die Mithilfe bei der Konfiszierung grenznaher Adelsbibliotheken angelegen sein, wobei er Tengnagel eine klare Rolle zuschrieb. ${ }^{15}$

Auch in anderen Zusammenhängen dokumentiert der Austausch zwischen Seyfried und Tengnagel die gegenseitige Positionsbestimmung der beiden Institutionen.

Seyfried konzipierte sein österreichisches Geschichtswerk in einer Weise, dass die in Zwettl vorhandenen Quellen - etwa die dort vorfindliche Version der Rudolfinischen Urkundensammlung ${ }^{16}$ - Element einer breiter angelegten österreichischen Geschichte werden konnte. Diese schloss Ungarn mit ein, und ein Hauptteil der Korrespondenz zwischen Seyfried und Tengnagel kreist um Erwähnungen der „Paiones” - eines antiken Stammes am Südbalkan bei griechischen Autoren. Seyfried war offensichtlich interessiert daran, eine Verbindung mit „Pannonien” herzustellen, er wollte möglichst genau die einschlägigen Zitate bei Homer, Pausanias, Herodot und Diodor zum Urtext zurückverfolgen und zugleich die spätere Kommentierung der Stellen nachvollziehen können. So kam es zu mehreren Anfragen nach Büchern und deren Ausleihe von Wien nach Zwettl.

Es lassen sich in diesem Prozess mehrere Dimensionen beobachten. Seyfried fragte Tengnagel zum einen nach spezifischen Büchern, die in

14 Nicolette Mout, Political and Religious Ideas of Netherlanders at the Court in Prague, in: Acta Historiae Neederlandicae, 9 (1976), 1-30, hier 26f. Klimannus an Tengnagel, 8. September 1621, ÖNB Cod. 9737s, 247r-248v. Es ist denkbar, dass auch die von Tengnagels Hand stammende „Designatio captivorum rebellium" (um 1620) in diesem Zusammenhang zu sehen ist: ÖNB, Cod. 7990, 55r-v.

16 Seyfried an Tengnagel, 30. August 1621, ÖNB Cod. 9737s, 244r-245v. Vgl. Lнотsку, Palatina (wie Anm. 9), 461f., wo auch die Editionsgeschichte des Stücks besprochen wird. 
Zwettl nicht vorhanden waren und die er gerne ausgeliehen hätte. ${ }^{17}$ In einem Fall erbat er die Abschrift einer konkreten Passage. ${ }^{18}$ Tengnagel wiederum notierte auf den Briefen Seyfrieds oft seine jeweilige Reaktion, und wenn der Stakkato-Stil seiner Dorsalvermerke noch der notizenhaften Textform geschuldet sein mag, so zeigte sich in den brieflichen Reaktionen des Zwettler Abtes, dass Tengnagel tatsächlich mit zunehmender Ungeduld die geliehenen Bücher zurückforderte (Авв. 2). ${ }^{19}$ Am Ende wurde die Ausleihe mit einem Schuldschein abgesichert. ${ }^{20}$

Seyfried ließ sich aber, zum zweiten, auch im Hinblick auf einschlägige Literatur und Quellen beraten. Wenn, so der Abt, Tengnagel eine Perle fände, die eingewoben den Schmuck des Annalenwerkes steigern könnte, so möge er sie ohne Neid mitteilen und werde lobende Erwähnung finden. ${ }^{21}$ Eine frühere bibliographische Sendung Tengnagels, gewissermaßen ein Handapparat an einschlägiger Bibliographie, war in Zwettl hingegen auf wenig Gegenliebe gestoßen: Die gesendeten Werke von Biondo, Zonara und Canisius halfen demnach nicht bei der Erarbeitung der frühen österreichischen Geschichte („incunabula historiae Austriacae”); und der „calvinistischen Krähe” Philipp Clüver sollten - mit einer Alliteration - alle Federn ausgerissen werden („Cluverio vero Calvinianae corniculae votum est ubique plumas detrahere”), worum sich Seyfried bereits bemüht hatte, als jener Noahs Sohn Ashkenaz zum Stammvater der Germanen hatte machen wollen. Dass solche Vorschläge dem ganzen historiographischen Vorhaben schaden, werde Tengnagel, kaum ohne Scham, einsehen müssen. ${ }^{22}$

17 Seyfried an Tengnagel, 2. November 1621, ÖNB Cold 9737s, 222r-223v: "libros hosce a te obtinuisse”; ebenso 20. April 1622, ebd. 267r-v.

Seyfried an Tengnagel, 22. September 1622, ÖNB Cod. 9737s, 275r-276v: „Similiter ex Zonara flagito mihi illum locum integre describe, quem mutile Hieronymus Wolphius in Annotationibus in Primam Olynthiacam Demosthenis citat his verbis $[\ldots] . "$

19 Etwa die Dorsalvermerke auf Cod. 9737s, 278v.

20 Seyfried an Tengnagel, 10. Dezember 1622, ÖNB Cod. 9737s, 282r-v.

21 Seyfried an Tengnagel, 16. November 1622, ÖNB Cod. 9737s, 277r-278v.

22 Seyfried an Tengnagel, 4. Dezember 1622, ÖNB Cod. 9737s, 279r-280v. Die Rede ist von Clüvers „Germania Antiqua”. 


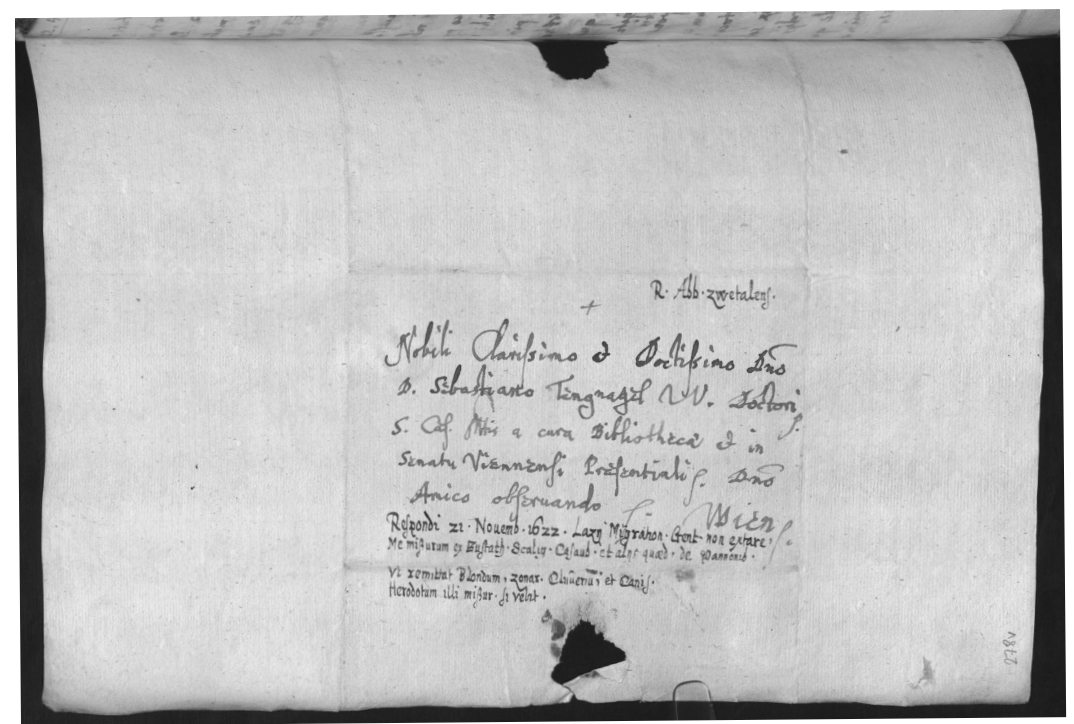

Abb. 2: Adresse und Vermerke auf der Rückseite des Briefes Seyfried an Tengnagel, 16. November 1622 (copyright: Österreichische Nationalbibliothek).

Es standen sich also mahnende Worte der Rechtgläubigkeit nebst latenter Drohgebärde gegenüber mit einem wie selbstverständlich konfessionsübergreifenden bibliographischen Gestus. Beide Gesprächspartner deuteten damit die jeweilige Schwäche des anderen an: Tengnagels angreifbare Rechtgläubigkeit auf der einen Seite, auf der anderen der fehlende fachliche und bibliographische Überblick aus der Perspektive einer ruralen Klosterbibliothek. ${ }^{23}$ Dieselbe Spannungslage prägte aber auch weitere Dimensionen des dokumentierten Austauschs im Herbst 1622. So sandte, drittens, der Zwettler Abt den Katalog seiner Bibliothek („index nostrae bibliothecae”) mit der Bitte, etwaige Inedita am Rand anzumerken und den Katalog

In schwächerer Form deutet auch Seyfrieds Bitte an Tengnagel, ihn bei der Suche nach einem neuen Gehilfen (a „Latinis litteris amanuensis”) zu unterstützen, in eine ähnliche Richtung; „Wenceslaus meus” kehrte in die Heimat zurück. Seyfried an Tengnagel. 4. Dezember 1622, ÖNB Cod. 9737s, 279r-280v. 
dann zurückzusenden. ${ }^{24}$ Es musste Seyfried bewusst sein, dass damit ein potentieller Klosterrat Einblick in die hauseigenen Bestände erhielt. Konstellationen dieser Art führten zwar erst unter Joseph II. dazu, dass es vielen österreichischen Klosterbibliotheken ähnlich erging wie nach 1620 den konfiszierten Bibliotheken protestantischer böhmischer Adeliger; doch bestand das grundsätzliche Spannungsverhältnis spätestens seit der Errichtung des Klosterrates durch Maximilian II.

Entlang einer ähnlichen Bruchlinie verlief, viertens, der Wunsch Seyfrieds, Tengnagel sein einmal fertiggestelltes Opus vorlegen zu können. ${ }^{25}$ Ziel dabei war die wissenschaftliche Absicherung, zugleich aber implizit auch jene institutionelle, die bereits bei der Approbation der "Arbor Aniciana” relevant gewesen war. Mit der Hofbibliothek verband sich bis weit ins 18. Jahrhundert eine informeller Anspruch, an der Deutung der österreichischen Geschichte beteiligt zu sein. Dass diese Geschichte stets auch habsburgisch war - in dem Sinn, dass auch von Mitgliedern der herrschenden Dynastie als Widmungsempfängern approbative Autorität ausgehen konnte -, das unterließ Seyfried ebenfalls nicht, beiläufig zu erwähnen. ${ }^{26}$ Das latente Ringen um Kompetenz und Deutungsmacht, das sich in der Korrespondenz spiegelt, hatte schließlich, fünftens, auch eine Dimension von materiellen Werten. Offenbar sandte Seyfried selbst Bücher nach Wien in der Hoffnung, Tengnagel möge sie als Tauschobjekte für einige der nach Zwettl übersandten Bücher akzeptieren. Zwar existieren keine direkten Äußerungen Tengnagels hierzu in den erhaltenen Briefen, doch macht der Tenor von Seyfrieds Antwort deutlich, wie diese ausgefallen sein mussten. Er selbst, so Seyfried, sei sich bewusst, dass die übersendeten Bücher nicht geeignet (,impares”) seien, um gegen irgendetwas eingetauscht zu werden, weshalb er unbeschadete 
Rückstellung versprach. ${ }^{27}$ Hier, anders als in konfessionellen Fragen, hat der Abt dem Hofbibliothekar nichts entgegenzuhalten.

Es ist in dem sehr eng gesteckten Rahmen dieses Beitrags nicht möglich, Seyfrieds Forschungstätigkeit weiter zu beleuchten. ${ }^{28}$ Ebensowenig kann der Versuch unternommen werden, die vorgestellten Ausschnitte im breiteren intellektuellen Profil Tengnagels zu kontextualisieren. ${ }^{29}$ Zweck der Ausführungen war vielmehr, auf zwei spezifische Spannungsfelder hinzuweisen, die sich, wie ausgeführt wurde, im Verhältnis der beiden Gelehrten spiegeln:

Bemerkenswert ist zum einen das methodisch sehr offene Verständnis von Geschichte, in dem die Grenzen zwischen klassischen, christlichen und heidnischen Altertümern mitunter nicht klar gezogen werden. Dies zeigt sich, wenn man Seyfrieds ethnographische Recherchen zur „ungarischen” Geschichte betrachtet und diese mit seinen anderen Arbeiten - der „Arbor Aniciana” ebenso wie den Forschungen zu Rudolf I. - vergleicht, ebenso mit Tengnagels Interesse für das Nachleben der griechischen Antike in den orientalischen Kulturen. Es existierte um 1600 kein eindeutiges methodisches Rüstzeug historischer Arbeit; ebenso wenig existierte für sie ein eindeutig bestimmter Gegenstand.

Zum zweiten spiegelt der Austausch zwischen Tengnagel und Seyfried auch eine latente Spannung zwischen den beiden jeweiligen Bibliotheken als Wissensorten. Hofbibliotheken besaßen um 1600 einen Wert, der zwar noch nicht an jenen der protostaatlichen Wissensspeicher künftiger Jahrhunderte heranreichte, jedoch sehr wohl in Verbindung mit tagespolitisch relevanter gelehrter Expertise und politischer Deutungsmacht stand. Die Klöster der vortridentinischen Orden auf der anderen Seite, etwa der Zisterzienser, konnten aufgrund Seyfried an Tengnagel, 22. September 1622, ÖNB Cod. 9737s, 275r-276v.

28 Vgl. das Verzeichnis ungedruckter historischer Arbeiten bei WatzL, Cistercienser (wie Anm. 3), 60.

29 Hülya Čelik-Chiara Petrolini, The Network of Court Librarian Sebastian Tengnagel and the Circulation of Oriental Manuscripts in the Early $17^{\text {th }}$ Century [im Druck in dem Band zur Tagung „Was wäre die Bibliothek ohne Bibliothekare?”, Wien 2020]. 
der von ihnen verwahrten Überlieferung oft ebenso Deutungsansprüche auf die Frühgeschichte eines Landes erheben und auf ein besonderes Verhältnis zur regierenden Dynastie verweisen, und sie taten dies in den Jahrzehnten nach 1600 dezidiert mit der Emphase der habsburgtreuen Rekatholisierung. Im Gegensatz zu vielen Bibliotheken protestantischer Adeliger (wie jener von Vöttau oder Wittingau), die eben diesem Prozess zum Opfer fielen, behaupteten sich Klöster wie Zwettl noch für rund zwei Jahrhunderte als Träger dezentraler Wissenskultur. Die langfristige Zukunft gehörte jedoch jenen Hofbibliotheken, die einmal zu Nationalbibliotheken werden sollten. Ihre relative Augenhöhe mit anderen Wissensorten des 16. und 17. Jahrhunderts muss heute bewusst mitgedacht werden, wenn man einen gelehrten Austausch wie den zwischen Johann Seyfried und Sebastian Tengnagel verstehen will. 
LES BIBLIOTHÈQUES ET L'ÉCONOMIE DES CONNAISSANCES Bibliotheken und die ÖKonomie des Wissens

$$
145^{\circ}-185^{\circ}
$$

Colloque international - Internationale Tagung 9-13 avril/April 2019 Sárospatak (Hongrie/Ungarn)

Édité par

Frédéric Barbier, István Monok et Andrea Seidler 


\section{L'Europe en réseaux}

Contribution à l'histoire de la culture écrite 1650-1918

Vernetztes Europa

Beiträge zur Kulturgeschichte des Buchwesens 1650-1918

Édité par

Frédéric Barbier, Marie-Elisabeth Ducreux, Matthias Middell, István Monok, Éva Ringh, Martin Svatoš

Volume VIII

École pratique des hautes études, Paris

École des hautes études en sciences sociales, Paris

Centre des hautes études, Leipzig

Bibliothèque nationale Széchényi, Budapest

Bibliothèque et centre d'information de l'Académie hongroise des sciences, Budapest 
LeS BIBLIOTHÈQUeS ET L'ÉCONOMIE DES CONNAISSANCES BibliotheKen und die ÖKonomie des Wissens

$$
145^{\circ}-185^{\circ}
$$

Colloque international - Internationale Tagung 9-13 avril/April 2019 Sárospatak (Hongrie/Ungarn)

Édité par

Frédéric Barbier, István Monok et Andrea Seidler

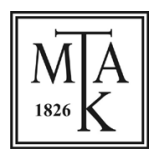

Magyar Tudományos Akadémia Könyvtár és Információs Központ Budapest 2020 
Mise en page

Viktória Vas

ISBN 978-963-7451-57-7

DOI 10.36820/SAROSPATAK.2020 
Préface

István MoNOK

Bibliothecae mutantur - Quare, quemadmodum et quid attinet?

Transformations de la composition thématique

des bibliothèques du Royaume de Hongrie aux $\mathrm{XV}^{\mathrm{E}}-\mathrm{XVI} \mathrm{I}^{\mathrm{e}}$ siècles....11

Marianne CARbonnier-Burkard

Les bibliothèques des Églises réformées françaises au XVII siècle.... 30

Max Engammare

De la bibliothèque de l'Académie de Calvin (1570) a la bibliothèque de l'Académie de Bèze (1612) à travers leur catalogue: Continuités et ruptures jusqu'au troisième catalogue de 1620........... 57

Róbert OLÁ́

Obsolescent Reformed Libraries in the seventeenth and eighteenth Century Carpathian Basin

Ádám Hegri

Moderner Zeitgeist - veraltete Lesestoffe. Bibliotheken reformierten Pfarrer um die Wende des 18. zum

19. Jahrhunderts im Königreich Ungarn

Petr MAšEK

Zierotin Library in Velké Losiny in Sixteenth and Seventeenth century. 136

Detlef HABERLAND

Schlesische Bibliotheken Zeichen der intellektuellen Vielfalt einer zentralen Bildungsregion in Europa 146

Thomas WaLLNIG

Sebastian Tengnagel und Johann Seyfried - Österreichische

Geschichtsschreibung zwischen Späthumanismus und

Gegenreformation 
Elisabeth EngL-Ursula RautenBerg

Christoph Jacob Trew - Bibliothek und Sammeln in der Gelehrtengemeinschaft der ersten Hälfte des 18. Jahrhunderts.

Helwi BLOM

Philosophie ou Commerce?

L'évolution des systèmes de classement bibliographique dans les catalogues de bibliothèques privées publies en France au XVIII ${ }^{\mathrm{e}}$ siècle.

Maria Luisa López-VIDrIero Abelló

Les meubles de la connaissance: façons de devenir sage à prix fixe.

Frédéric BARbier

Distinction, récréation, identité: la trajectoire des « romans» en France sous d'Ancien Régime.

\section{Andrea SeIdler}

Die praktische Bedeutung ungarischer Sammlungen und Bibliotheken für führende Gelehrte des Königreichs Ungarn im späten 18. Jahrhundert am Beispiel des Jesuiten Georg Pray (1723-1801).

\section{Olga Granasztói}

Se divertir: les enseignements de la bibliothèque d'une femme aristocrate hongroise à la fin du XVIII siècle

Christophe Didier

La naissance du théâtre „des boulevards”, ou Comment la banlieue entre en bibliothèque (1780-1830).

\section{Andrea De Pasquale}

La nascita delle riserve di libri antichi in Italia

Index des noms de personne et de lieu..... 